\section{Statin therapy for prevention of vascular events should be offered to lower-risk subjects}

The heart protection study - a randomized, placebo-controlled trial of 20,536 men and women in the UK aged $40-80$ years and at increased risk of vascular eventsdemonstrated the effectiveness of simvastatin ( $40 \mathrm{mg}$ per day for 5 years) in reducing the rates of major vascular events. The authors have now used these data to predict whether lifetime use of the drug is cost-effective in a range of different age and risk groups.

An internally validated model was developed and used to predict the yearly rates of death (from both vascular and nonvascular causes) and nonfatal major and other vascular events. Projected hospital costs associated with these events were also calculated.

Estimated life-years gained from taking simvastatin ranged from $0.64(95 \% \mathrm{Cl} 0.31-$ $0.98)$ for those over 70 years and in the lowest risk group (12\% risk of a major vascular event in 5 years) to 2.49 (95\% Cl 1.55-3.36) for individuals aged $40-49$ years and at greatest risk of a major event ( $42 \%$ over 5 years). At the April 2005 price of UK£4.87 (€7, US\$9) per 28-day pack of generic simvastatin, extrapolation beyond the 5-year treatment period showed lifetime adherence to be less costly than hospitalization associated with vascular events in all individuals except those 70 years and above in the lowest risk group. Further extrapolation indicated that the drug would be cost-effective (i.e. cost less than $£ 2,500$ per life-year) when administered to people as young as 35 years and as old as 85 years at very low risk ( $1 \%$ annually) of a major vascular event.

Original article Heart Protection Study Collaborative Group (2006) Lifetime cost effectiveness of simvastatin in a range of risk groups and age groups derived from a randomized trial of 20536 people. BMJ [doi:10.1136/ bmj.38993.731725.BE]

\section{Carotid plaque surface irregularities increase risk of ischemic stroke}

The presence of carotid stenosis is a wellestablished risk factor for ischemic stroke. There is some evidence that plaque surface morphology is an additional indicator of stroke risk; however, data from large population-based studies are lacking.

To examine the association between plaque surface morphology and risk of ischemic stroke, researchers from the Neurological Institute at Columbia University, New York, NY, conducted an analysis in the multiethnic (51.4\% Hispanic, $24.4 \%$ black, $21.8 \%$ white, $2.4 \%$ other) prospective Northern Manhattan Study cohort. High-resolution B-mode ultrasonography was used to examine carotid plaque surface characteristics in 1,939 individuals (mean age 68.6 years) with no history of stroke. Overall, 1,091 (56.4\%) individuals exhibited carotid plaque, with an irregular plaque surface being documented in 107 (5.5\%) subjects. After a mean follow-up of 6.2 years, 69 (3.6\%) of the study population had been diagnosed with ischemic stroke. Individuals with plaque surface irregularities had an $8.5 \%$ unadjusted cumulative 5-year risk of ischemic stoke, compared with $3.0 \%$ for those with regular plaque and $1.3 \%$ for those with no plaque. Following adjustment for age, sex, ethnicity, traditional vascular risk factors, degree of stenosis and plaque thickness, the presence of irregular plaque increased the risk of ischemic stroke fourfold compared with no plaque. Irregular plaque was also predictive of a combined vascular outcome comprising ischemic stroke, myocardial infarction and vascular death. On the basis of these results, the authors conclude that plaque surface irregularity could be a useful marker of vascular risk in addition to conventional risk factors.

Original article Prabhakaran S et al. (2006) Carotid plaque surface irregularity predicts ischemic stroke: the Northern Manhattan Study. Stroke 37: 2696-2701 